\title{
Dielectric relaxation studies of binary mixture of $\beta$-picoline and methanol using time domain reflectometry at different temperatures
}

\author{
C. M. Trivedi*, V. A. Rana*, , P. G. Hudge ${ }^{\dagger}$ and A. C. Kumbharkhane \\ *Department of Physics, Gujarat University \\ Ahmedabad-380009, Gujarat, India \\ ${ }^{\dagger}$ Allied Sciences Department, MKSSS's Cummins College of Engineering \\ Pune, Maharashtra, India \\ ${ }^{\dagger}$ School of Physical Sciences, Swami Ramanand Teerth \\ Marathawada University, Nanded, Maharashtra, India \\ §varana@gujaratuniversity.ac.in
}

Received 15 March 2016; Revised 3 June 2016; Accepted 7 July 2016; Published 22 August 2016

\begin{abstract}
Complex permittivity spectra of binary mixtures of varying concentrations of $\beta$-picoline and Methanol $(\mathrm{MeOH})$ have been obtained using time domain reflectometry (TDR) technique over frequency range $10 \mathrm{MHz}$ to $25 \mathrm{GHz}$ at $283.15,288.15,293.15$ and $298.15 \mathrm{~K}$ temperatures. The dielectric relaxation parameters namely static permittivity $\left(\varepsilon_{0}\right)$, high frequency limit permittivity $\left(\varepsilon_{\infty 1}\right)$ and the relaxation time $(\tau)$ were determined by fitting complex permittivity data to the single Debye/Cole-Davidson model. Complex nonlinear least square (CNLS) fitting procedure was carried out using LEVMW software. The excess permittivity $\left(\varepsilon_{0}^{\mathrm{E}}\right)$ and the excess inverse relaxation time $(1 / \tau)^{E}$ which contain information regarding molecular structure and interaction between polar-polar liquids were also determined. From the experimental data, parameters such as effective Kirkwood correlation factor $\left(g^{\text {eff }}\right)$, Bruggeman factor $\left(f_{\mathrm{B}}\right)$ and some thermo dynamical parameters have been calculated. Excess parameters were fitted to the Redlich-Kister polynomial equation. The values of static permittivity and relaxation time increase nonlinearly with increase in the mol-fraction of $\mathrm{MeOH}$ at all temperatures. The values of excess static permittivity $\left(\varepsilon_{0}{ }^{E}\right)$ and the excess inverse relaxation time $(1 / \tau)^{\mathrm{E}}$ are negative for the studied $\beta$-picoline $-\mathrm{MeOH}$ system at all temperatures.
\end{abstract}

Keywords: Complex permittivity; relaxation time; time domain reflectometry; CNLS fitting; Redlich-Kister equation; $\beta$-picoline.

\section{Introduction}

Dielectric relaxation studies of polar liquids involve measurements of complex permittivity (dielectric constant and dielectric loss) over a range of frequency and temperature. Measured complex permittivity data can be used to find dielectric relaxation time, distribution parameter, static permittivity and thermodynamical parameters. These parameters provide useful information about the molecular size, shape, inter and intramolecular interactions and molecular structures. Time Domain Reflectometry (TDR) has widely been used by many researchers to measure the complex permittivity of polar liquids and their binary mixtures. ${ }^{1-6}$

Recently we reported results of our study of dielectric relaxation in the binary mixtures of $\alpha$-picoline and Methanol $(\mathrm{MeOH})$ at different temperatures using Time Domain Reflectometry (TDR) technique. ${ }^{7}$ In continuation of this work, study of dielectric relaxation behavior of binary mixtures of $\beta$-picoline and methanol $(\mathrm{MeOH})$ at different temperatures using TDR technique was undertaken and the results are reported in this paper. $\alpha$-picoline and $\beta$-picoline are the isomers of methyl pyridine with formula $\mathrm{C}_{6} \mathrm{H}_{7} \mathrm{~N}$. At room temperature they are colorless liquids. They are used as precursor to pyridine derivatives and have wide range of applications in pharmaceutical and agricultural industries. The other component considered for the binary mixture system is the lower primary alcohol $\mathrm{MeOH}$. Alcohols play an important role in many chemical reactions on account of their ability to undergo self-association with manifold internal structures. ${ }^{8}$ They are widely used in industries and science as reagents, solvents and fuels.

Due to the presence of lone pair of electron on the nitrogen atom site of picoline and proton donor sites in the alcohol molecules an $\mathrm{O}-\mathrm{H}-\mathrm{N}$ type of proton donoracceptor linkage is expected in the mixtures of picoline and $\mathrm{MeOH}$. Dielectric relaxation parameters of the like and unlike molecules are considerably affected by the H-bond interaction. Thus it is thought that this study will provide a good scope to analyze dielectric data obtained using TDR technique for binary mixture system of $\beta$-picoline and $\mathrm{MeOH}$. Not only that but it will also provide information about the molecular interaction taking place between the components of the binary mixture system. Literature survey suggests that very few researchers have studied dielectric behavior of picoline in its pure state or in a mixed state with other

This is an Open Access article published by World Scientific Publishing Company. It is distributed under the terms of the Creative Commons Attribution 4.0 (CC-BY) License. Further distribution of this work is permitted, provided the original work is properly cited. 


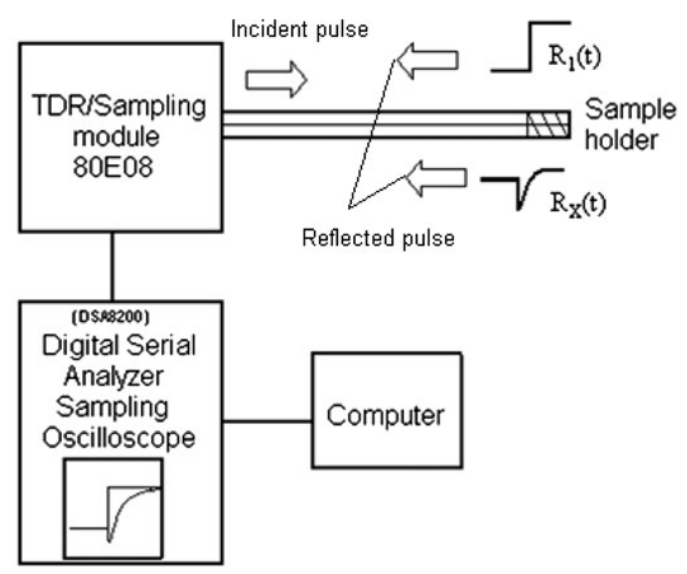

(a)

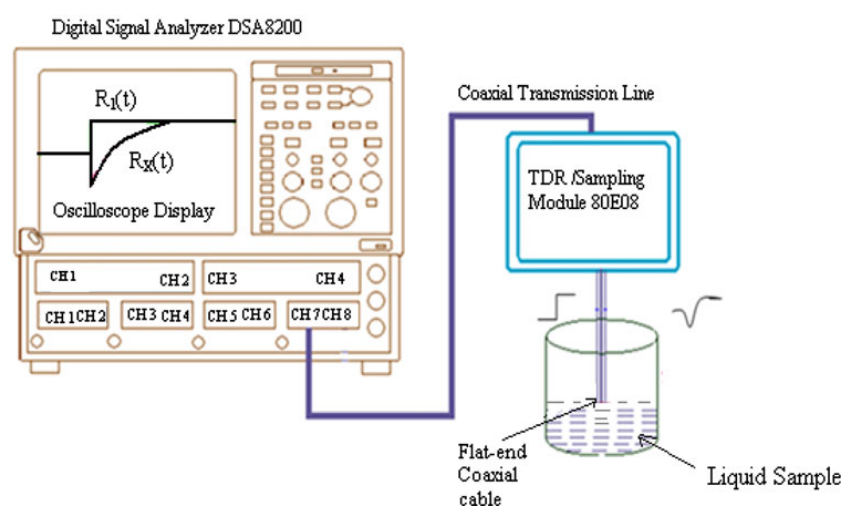

(b)

Fig. 1. Block diagram of TDR.

polar/nonpolar solvents, through physical $^{9-11}$ or dielectric methods. ${ }^{12,13}$

Aim of the present investigation is to work out a relaxation model for describing the broadband complex permittivity spectra $\varepsilon^{*}(\omega)=\varepsilon^{\prime}(\omega)-j \varepsilon^{\prime \prime}(\omega)$ of $\beta$-picoline-MeOH mixtures with various compositions over the frequency range $10 \mathrm{MHz}$ to $25 \mathrm{GHz}$ at four different temperatures: $283.15 \mathrm{~K}$, $288.15 \mathrm{~K}, 293.15 \mathrm{~K}, 298.15 \mathrm{~K}$ and to gain information about the orientational dynamics and molecular interaction among the molecules of the binary liquid mixture system.

\section{Experimental Procedure}

$\mathrm{MeOH}$ (AR grade) and $\beta$-picoline (AR grade) with $99 \%$ purity were procured from S. d. Fine chem Limited (India). Binary mixtures of $\beta$-picoline and $\mathrm{MeOH}$ were prepared at nine concentrations by volume at room temperature. Using following formula these concentrations were converted into the mol fraction of $\mathrm{MeOH}$ in $\beta$-picoline.

$$
X_{1}=\frac{\rho_{1} \times \frac{v_{1}}{M_{1}}}{\rho_{1} \times \frac{v_{1}}{M_{1}}+\rho_{2} \times \frac{v_{2}}{M_{2}}},
$$

where $M$ is molecular weight, $v$ is volume and $\rho$ is literature value of density. 1 represents $\mathrm{MeOH}$ and 2 represent $\beta$-picoline. The maximum error in mole fraction is $\pm 0.1 \%$.

The complex permittivity spectra of $\beta$-picoline, $\mathrm{MeOH}$ and their mixtures were obtained by TDR technique. The Tektronics model no. DSA8200 Digital serial analyzer sampling oscilloscope along with the sampling module 80E08 has been used and this module provides accurate oscilloscope measurement with user selectable bandwidth of 20 or $30 \mathrm{GHz}$. The block diagram and experimental setup of TDR is shown in Fig. 1. The coaxial cable semi rigid, copper, EZ_86/M17 (Huber+ Suhner Electronics pvt. Ltd.) with flat end was used. The outer diameter of cable is $2.2 \mathrm{~mm}$. Sampling oscilloscope monitors changes in step pulse after reflection from the end of line. Reflected pulse without sample $R_{1}(t)$ and with sample $R_{X}(t)$ were recorded in time window of $2 \mathrm{~ns}$ and digitized in 2000 points in the memory of the oscilloscope and transferred to the computer. These recorded pulses are added $\left[q(t)=R_{1}(t)+R_{X}(t)\right]$ and subtracted $\left[p(t)=R_{1}(t)-R_{X}(t)\right]$. Further the Fourier transformation of $p(t)$ and $q(t)$ was obtained by Shannon ${ }^{14}$ and Samulon ${ }^{15}$ methods respectively, for the frequency range $10 \mathrm{MHz}$ to $25 \mathrm{GHz}$.

Figure 2 shows the complex permittivity spectra of $\beta$-picoline, $\mathrm{MeOH}$ and their mixtures at $283.15 \mathrm{~K}$ temperature, which shows a systematic variation of $\varepsilon^{\prime}$ and $\varepsilon^{\prime \prime}$ with frequency. Permittivity at optical frequency, $\varepsilon_{\infty}$ for pure liquids and $\varepsilon_{\infty \mathrm{m}}$ for the binary mixtures were taken as square of the refractive index, $n_{\mathrm{D}}$, which was measured with an Abbe refractometer at wavelength of sodium-D light. The standard uncertainty of measurement of the refractometer is $0.001 \%$.

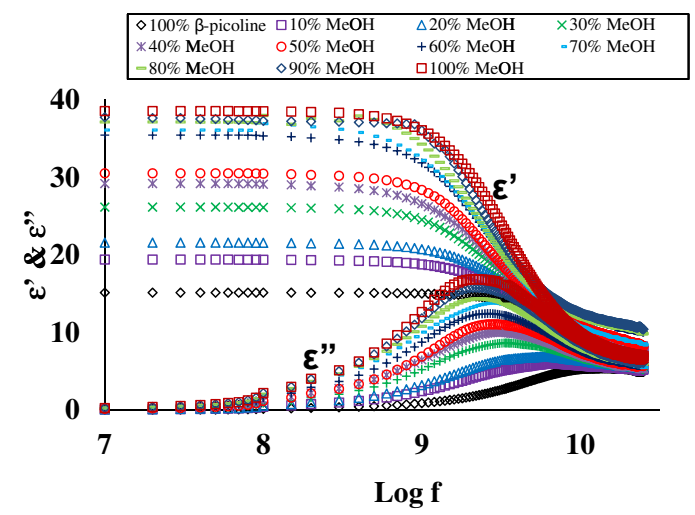

Fig. 2. Complex permittivity spectra $\left[\varepsilon^{*}(\omega)=\varepsilon^{\prime}(\omega)-j \varepsilon^{\prime \prime}(\omega)\right]$ for $\beta$-picoline $+\mathrm{MeOH}$ at $283.15 \mathrm{~K}$. 


\section{Data Analysis}

The dielectric constant and loss were initially plotted in complex plane. Spectra for the samples were found to display a single dispersion in the considered frequency range. It was observed that the first dispersion encountered was quantitatively described by a semicircle with intercepts on the real axis at $\varepsilon^{\prime}=\varepsilon_{0}$ and $\varepsilon^{\prime}=\varepsilon_{\infty 1}$, representing the limiting low and high frequency permittivity for this dispersion. The choice of the relaxation model depends on the frequency range of measurement and on the quality of the data. ${ }^{16}$ Since the complex permittivity measurements in the present study are in the frequency range of $10 \mathrm{MHz}-25 \mathrm{GHz}$, we have applied Cole-Davidson/Debye function, ${ }^{17,18}$

$$
\varepsilon^{*}=\varepsilon_{\infty 1}+\frac{\varepsilon_{0}-\varepsilon_{\infty 1}}{[1+(j \omega \tau)]^{\beta}}
$$

to obtain relaxation parameters for the main relaxation process. In this equation, $\varepsilon_{0}$ and $\varepsilon_{\infty 1}$ represent the limiting low and high frequency permittivity, $\tau$ is the relaxation time due to main relaxation process and $\beta$ is the asymmetric distribution parameter. Equation (2) reaches to Debye function when $\beta$ equals to one. CNLS (Complex Non linear Least Square) fitting procedure was carried out using LEVMW. ${ }^{19}$ CNLS fitting takes both the real and imaginary parts of the complex permittivity into the objective function simultaneously to ensure the complete fit. ${ }^{20}$

The Kirkwood correlation factor $(g)$ is a parameter that supplies information regarding the orientation of the electric dipoles in polar liquids. For pure liquids it is given by ${ }^{21,22}$

$$
\frac{4 \pi N_{A} \mu^{2} d}{9 k T M} g=\frac{\left(\varepsilon_{0}-\varepsilon_{\infty}\right)\left(2 \varepsilon_{0}-\varepsilon_{\infty}\right)}{\varepsilon_{0}\left(\varepsilon_{\infty}+2\right)^{2}},
$$

where $\rho$ is the density of liquid, $M$ is the molecular weight, $k$ is the Boltzmann constant, $N$ is the Avogadro's number and $\mu$ is the dipole moment of polar molecule in gas phase. $\varepsilon_{\infty}$ is taken as square of the refractive index.

For the mixtures of two polar liquids the effective averaged angular Kirkwood correlation factor $\left(g^{\text {eff }}\right)$ of two different molecules were evaluated from the modified Kirkwood equation for the binary mixture ${ }^{21,22}$

$$
\begin{gathered}
\frac{4 \pi N}{9 k T}\left(\frac{\mu_{1}^{2} d_{1}}{M_{1}} x_{1}+\frac{\mu_{2}^{2} d_{2}}{M_{2}} x_{2}\right) g^{\mathrm{eff}} \\
=\frac{\left(\varepsilon_{m}-\varepsilon_{\infty m}\right)\left(2 \varepsilon_{m}-\varepsilon_{\infty m}\right)}{\varepsilon_{m}\left(\varepsilon_{\infty m+2}\right)^{2}},
\end{gathered}
$$

where $X$ is the mole fraction of the liquid and subscripts $m, 1$ and 2 represent the mixture, $\mathrm{MeOH}$ and $\beta$-Picoline respectively. $\varepsilon_{m}$ is the static permittivity of the mixture, $\varepsilon_{\infty m}$ is the permittivity at optical frequency of the mixtures.

Excess static permittivity $\left(\varepsilon_{0}^{E}\right)$ was calculated using the relation ${ }^{23}$,

$$
\varepsilon_{0}^{E}=\varepsilon_{0 m}-\left(\varepsilon_{1} X_{1}+\varepsilon_{2} X_{2}\right) .
$$

Here $\varepsilon_{1}, \varepsilon_{2}$ are relative permittivity and $x_{1}$ and $x_{2}$ are mole fractions of components 1 and 2, respectively, and $\varepsilon_{m}$ is the static permittivity of mixture.

Excess inverse relaxation time of $\left(\frac{1}{\tau}\right)^{E}$ was calculated using the relation, ${ }^{24}$

$$
\left(\frac{1}{\tau}\right)^{E}=\left(\frac{1}{\tau}\right)_{m}-\left[\left(\frac{1}{\tau}\right)_{1} X_{1}+\left(\frac{1}{\tau}\right)_{2} X_{2}\right],
$$

where, $\left(\frac{1}{\tau}\right)_{m}$ is inverse relaxation time of mixture, and $\left(\frac{1}{\tau}\right)_{1}$ and $\left(\frac{1}{\tau}\right)_{2}$ are inverse relaxation time of components 1 and 2 .

The hetero (A-B) interaction between the components of the mixture can also be obtained from the Bruggeman equation. ${ }^{25,26}$ The Bruggeman factor $f_{\mathrm{B}}$ is related to the volume fraction of one of the components as:

$$
f_{B}=\left(\frac{\varepsilon_{m}-\varepsilon_{2}}{\varepsilon_{1}-\varepsilon_{2}}\right)\left(\frac{\varepsilon_{1}}{\varepsilon_{m}}\right)^{\frac{1}{3}}=\left(1-\phi_{2}\right) .
$$

$f_{B}$ may vary from one to zero as $\phi_{2}$ varies from zero to one. If there is no interaction between the components in the mixture, then $f_{B}$ should have a linear relationship with $\varphi_{2}$. If there is interaction between the components in the mixture, then Eq. (7) is modified as

$$
\left(\frac{\varepsilon_{m}-\varepsilon_{2}}{\varepsilon_{1}-\varepsilon_{2}}\right)\left(\frac{\varepsilon_{1}}{\varepsilon_{m}}\right)^{\frac{1}{3}}=1-\left[a-(a-1) \phi_{2}\right] \phi_{2},
$$

where ' $a$ ' is the Bruggeman parameter, which gives valuable information about heterogeneous interaction between the molecules. Using least square fitting method, the value of ' $a$ ' was determined. It is assumed that the volume fraction of the solute in the mixture is modified by a factor of $\left[a-(a-1) \phi_{2}\right] \phi_{2}$.

The energy parameters for dielectric relaxation process $\left(\Delta F_{E}, \Delta H_{E}\right.$ and $\left.\Delta S_{E}\right)$ have been evaluated employing Eyring's equation, ${ }^{27}$

$$
\begin{gathered}
\tau=\frac{h}{k T} \exp \left(\frac{\Delta F_{E}}{R T}\right), \\
\Delta F_{E}=\Delta H_{E}-T \Delta S_{E},
\end{gathered}
$$

where $h$ is plank's constant, $k$ is Boltzmann constant, $R$ is the gas constant, $T$ is temperature, $\Delta F_{E}$ is the molar free energy of activation, $\Delta H_{E}$ is the molar free enthalpy of activation and $\Delta S_{E}$ is the molar free entropy of activation for dielectric relaxation.

The variations of, excess static permittivity and excess inverse relaxation time with composition are expressed by Redlich-Kister ${ }^{28}$ polynomial equation,

$$
Q=x_{1} x_{2} \sum_{r=0}^{n} a_{r}\left(x_{2}-x_{1}\right)^{r},
$$

where $Q$ refers all excess parameters. 
The standard deviations $\sigma(Q)$ were defined as

$$
\sigma(Q)=\left|\frac{\Phi_{\min }}{P-n}\right|^{0.5},
$$

where $P$ and $n$ are the experimental points and parameters, respectively. $\Phi_{\min }$ is the minimum value of the objective function $\Phi$ defined as,

$$
\Phi=\sum_{k=1}^{N}\left(n_{k}\right)^{2},
$$

where $n_{k}=Q_{\text {cal }}--Q \cdot Q$ is the experimental value and $Q_{\text {cal }}$ is evaluated using Eq. (11).

\section{Results and Discussion}

Typical complex plane plots for pure $\beta$-picoline, $\mathrm{MeOH}$ and their binary mixtures of $30-70 \%, 50-50 \%$ and $40-60 \%$ concentrations at $288.15 \mathrm{~K}$ temperature are shown in Fig. 3. From the Figure, it can be seen that all the spectra display a single dispersion due to main relaxation process in the considered frequency range. The plot of $\beta$-picoline exhibits the principle dispersion of Debye type, whereas its mixture with $\mathrm{MeOH}$ and pure $\mathrm{MeOH}$ exhibit Cole- Davidson (C-D) type of relaxation behavior. Therefore we have determined dispersion parameters for $\beta$-picoline using Debye function, and for $\mathrm{MeOH}$ and its mixture with $\beta$-picoline at all the concentrations using $\mathrm{C}-\mathrm{D}$ function. Complex plane plots of $\alpha$-picoline-MeOH mixtures exhibited similar behavior. ${ }^{7}$

Static permittivity values obtained using the fitting procedures are plotted as a function of mole fraction of $\mathrm{MeOH}$ in Fig. 4. As can be seen from Fig. 4, with increasing fraction of $\mathrm{MeOH}$, static permittivity increases non-linearly. This confirms that intermolecular association is taking place in the system and possibly $\mathrm{O}-\mathrm{H}-\mathrm{N}$ type of hydrogen bonded structures between $\beta$-picoline and $\mathrm{MeOH}$ molecules are formed. The observed value of static permittivity of pure $\beta$-picoline in present investigation is higher than our earlier reported value of pure $\alpha$-picoline at all temperatures. ${ }^{7}$ The larger value of static permittivity of $\beta$-picoline is may be due

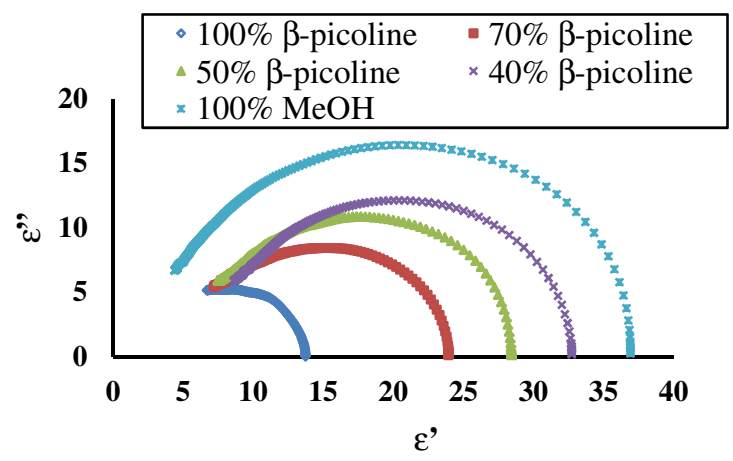

Fig. 3. Complex plane plots for binary mixtures of $\beta$-picoline, $\mathrm{MeOH}$ and mixture of $\beta$-Picoline-MeOH at $288.15 \mathrm{~K}$.

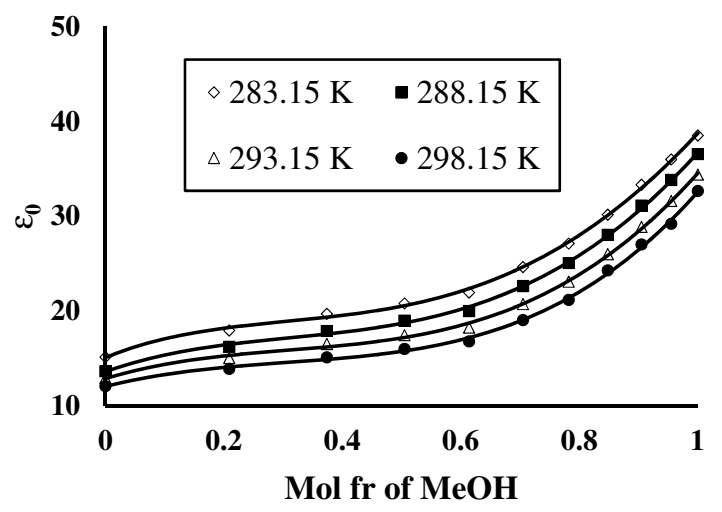

Fig. 4. Plot of static permittivity $\left(\varepsilon_{0}\right)$ against $\mathrm{Mol}$ fr of MeOH. (-) solid line shows the polynomial fitted curve.

to the change of $-\mathrm{CH}_{3}$ (methyl group) position in the pyridine ring structure from ortho to meta position. Similar behavior was observed by Rana et al. ${ }^{1}$ in static permittivity values of chloro substituted anilines.

The excess static permittivity value and its variation with mol fraction of $\mathrm{MeOH}$ at different temperatures is shown in Fig. 5. From the figure it can be seen that $\varepsilon_{0}^{E}$ is negative for all concentrations of $\mathrm{MeOH}$ in the mixture at all temperatures. This indicates that the molecules of the mixtures may form multimer structures via hydrogen bonding in such a way that the effective dipole moment gets reduced. It can also be seen from Fig. 6 that $\varepsilon_{0}^{E}$ is maximum negative at around 0.614 mole fraction of $\mathrm{MeOH}$. Large magnitude values of $\varepsilon_{0}^{E}$ of $\beta$-picoline-MeOH mixture confirms that $\beta$-picoline molecules acts as structure breakers for hydrogen bonded $\mathrm{MeOH}$ molecules with decrease in total number of parallel aligned dipoles. The magnitude values of $\varepsilon_{0}^{E}$ can be ordered with temperature variation as $\varepsilon_{0}^{E}(283.15)<\varepsilon_{0}^{E}(288.15)<$ $\varepsilon_{0}^{E}(293.15)<\varepsilon_{0}^{E}(298.15)$. This clearly suggest that hydrogen bonded molecular networks are more effectively dissociated with increase in temperature.

The plot of refractive index against mole fraction of $\mathrm{MeOH}$ at different temperatures is shown in Fig. 6. From

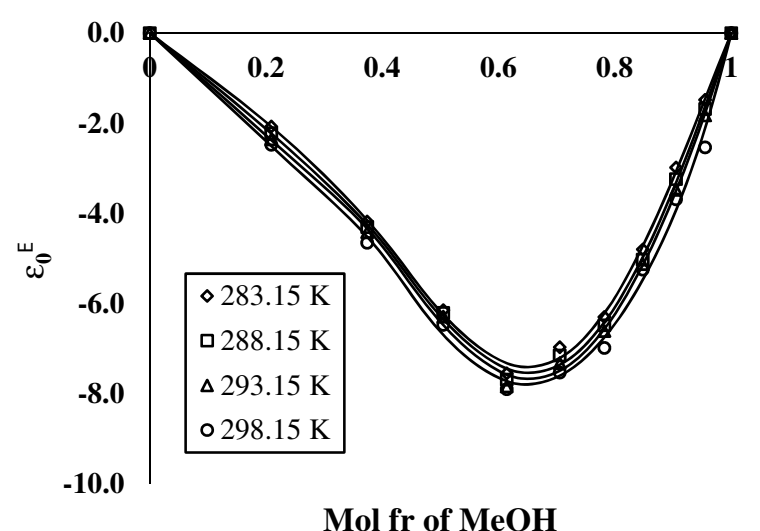

Fig. 5. Plot of excess static permittivity $\left(\varepsilon_{0}^{E}\right)$ against Mol fr of $\mathrm{MeOH}$. (-) show the fitted line of Redlich-Kister equation. 


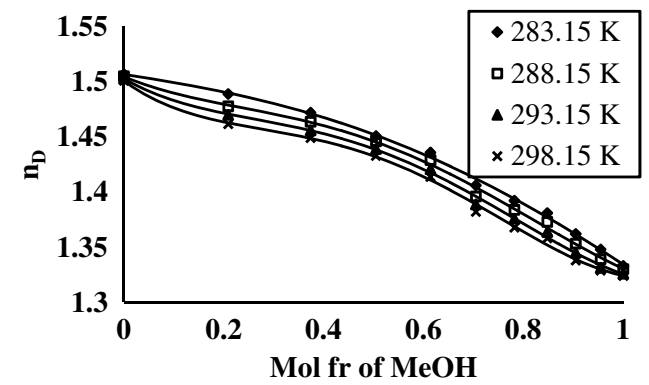

Fig. 6. Plot of refractive index $n_{\mathrm{D}}$ against Mol fr of MeOH. (一) solid line shows the polynomial fitted curve.

the figure it can be seen that refractive index of $\beta$ - picoline + $\mathrm{MeOH}$ mixtures decreases nonlinearly with mole fraction of $\mathrm{MeOH}$. For the visible range of frequencies, the electric field component of electromagnetic wave interacts with the electron cloud surrounding each atom or molecule within its path in such a way as to induce electronic polarization. As a consequence of this polarization light waves are retarded in velocity as they pass through the medium. Since the retardation of electromagnetic radiation results from electronic polarization, the size of the constituent atoms or molecules has a considerable influence on the magnitude of this effectgenerally, the larger an atom or molecule, the greater will be the electronic polarization and slower the velocity or higher the refractive index. ${ }^{29}$ Refractive index of pure $\beta$-picoline and $\mathrm{MeOH}$ are 1.507 and 1.333 , respectively at $283.15 \mathrm{~K}$ temperature. This clearly suggests that size of $\beta$-picoline molecule is larger than $\mathrm{MeOH}$. Furthermore, variation of refractive index as a function of mole fraction of $\mathrm{MeOH}$ suggests that the electronic polarization of the molecules in the mixed state is influenced by the $\mathrm{H}$-bonded interaction between $\beta$-picoline and $\mathrm{MeOH}$. The increase in temperature causes increase in inter-atomic spacing and this result in the decrease in refractive index with rise in temperatures at all mixture concentrations. High frequency permittivity $\varepsilon_{\infty 1}$, obtained by fitting experimental data to Eq. (2) were found to be different than the experimentally determined permittivity at optical frequency $\varepsilon_{\infty}$. In case of $\mathrm{MeOH}, \varepsilon_{\infty 1}$ is markedly different from $\varepsilon_{\infty}$ value $\left(\varepsilon_{\infty 1}-\varepsilon_{\infty}=2.48\right.$ at $\left.298.15 \mathrm{~K}\right)$, which confirms the presence of further absorption in the millimeter and infrared region. ${ }^{7}$ In case of pure $\beta$-picoline, a small difference in the values of $\varepsilon_{\infty 1}$ and $\varepsilon_{\infty}(0.65$ at $298.15 \mathrm{~K})$ is found. This indicates the presence of further absorption of very small amplitude in the $\mathrm{mm} / \mathrm{infrared}$ region and it can be due to the rotational orientation of the $-\mathrm{CH}_{3}$ group. When $\mathrm{MeOH}$ is mixed with $\beta$-picoline, main relaxation process of component molecules fuse in to one relaxation process and overlapping of relaxation processes seems to take place. Degree of overlapping varies with variation in the concentration of the constituents of the mixtures, causing increase in the difference $\varepsilon_{\infty 1}-\varepsilon_{\infty}$ values with increase in concentration of $\mathrm{MeOH}$.

The relaxation time value for pure $\beta$-picoline is $11.61 \mathrm{ps}$ at $293.15 \mathrm{~K}$, while the relaxation time of pure $\alpha$-picoline is
$10.61 \mathrm{ps}$ at the same temperature. ${ }^{7}$ Higher value of relaxation time of $\beta$-picoline than $\alpha$-picoline is in agreement with the higher value of viscosity of $\beta$-picoline $(0.9459 \mathrm{mPa} \cdot \mathrm{s}$ at $293.15 \mathrm{~K})^{30}$ than $\alpha$-picoline $(0.8102 \mathrm{mPa} . \mathrm{s}$ at $293.15 \mathrm{~K}){ }^{31}$ Smaller the viscosity of a liquid more is the ease of rotational orientation of the molecule and smaller is the relaxation time. The $\tau$ value of $\beta$-picoline is much smaller than $\mathrm{MeOH}$, which clearly suggests the associative and nonassociative nature of $\mathrm{MeOH}$ and $\beta$-picoline, respectively. The relaxation time decreases with increase in temperature at all concentrations. This is may be due to the increase in the thermal agitation and partly due to decrease in the viscosity with rise in temperature.

The variation of $(1 / \tau)^{E}$ with mole fraction of $\mathrm{MeOH}$ at $283.15,288.15,293.15$ and $298.15 \mathrm{~K}$ temperatures is shown in Fig. 7. From the figure, it can be seen that $(1 / \tau)^{E}$ is negative for the entire concentration range of the mixture. The negative $(1 / \tau)^{E}$ indicating formation of slowly rotating structures. The evaluated values of excess static permittivity and excess inverse relaxation time were fitted to RedlichKister polynomial. ${ }^{28}$ The coefficient $\mathrm{a}_{j}$ for $j=0$ to 4 are listed in Table 1.

Figure 8 shows the variation of $g^{\text {eff }}$ for the $\beta$ - picoline + $\mathrm{MeOH}$ mixture at different temperatures. For pure $\beta$ - picoline, $\mathrm{MeOH}$ and entire concentration range of their mixtures, $g^{\text {eff }}$ values are greater than unity, which indicates that dipolar alignment favor parallel orientations. It can also be seen from Figure that, the $g^{\text {eff }}$ vary with change in temperature, indicating that temperature change produces appreciable structural change. The determined values of Bruggeman factor $\left(f_{\mathrm{B}}\right)$ are shown in Fig. 9, which shows a nonlinear relation between $f_{\mathrm{B}}$ and volume fraction of $\mathrm{MeOH}$ at all temperatures. The Bruggemann parameter ' $a$ ' is a dielectric parameter, which provides information about the interaction between components in the mixture in terms of volume changes. The values of fitting parameter ' $a$ ' for $\beta$-picoline$\mathrm{MeOH}$ mixtures along with standard error and correction coefficients are presented in Table 2. At temperatures 283.15

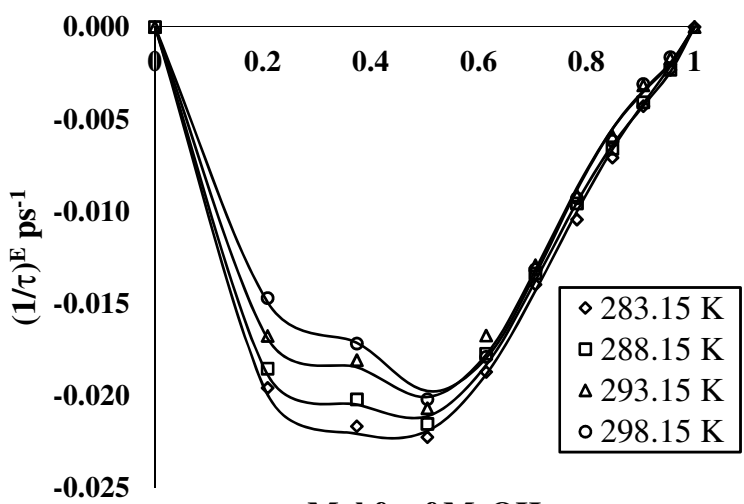

Mol fr of $\mathrm{MeOH}$

Fig. 7. Plot of excess inverse relaxation time $(1 / \tau)^{E}$ against Mol $\mathrm{fr}$ of MeOH. (-) show the fitted line of Redlich-Kister equation. 
Table 1. Parameters of Redlich-Kister equation for the mathematical representation of deviation in excess functions for $\beta$-picoline $+\mathrm{MeOH}$.

\begin{tabular}{lcccccccc}
\hline Excess properties & Temp. & \multicolumn{1}{c}{$a_{0}$} & $a_{1}$ & $a_{2}$ & \multicolumn{1}{c}{$a_{3}$} & $a_{4}$ & $\sigma$ & $\delta$ \\
\hline$\varepsilon_{0}^{E}$ & $283.15 \mathrm{~K}$ & -24.731 & 28.869 & 1.992 & -24.361 & -4.227 & 0.153 & 0.999 \\
& $288.15 \mathrm{~K}$ & -25.143 & 29.064 & 1.423 & -24.141 & -8.508 & 0.154 & 0.999 \\
& $293.15 \mathrm{~K}$ & -25.652 & 29.491 & 2.147 & -25.319 & -14.064 & 0.154 & 0.999 \\
& $298.15 \mathrm{~K}$ & -26.532 & 28.920 & 6.184 & -22.564 & -27.210 & 0.238 & 0.997 \\
$(1 / \tau)^{E}$ & $283.15 \mathrm{~K}$ & -0.088 & -0.027 & 0.034 & -0.082 & -0.111 & 0.000 & 1.000 \\
& $288.15 \mathrm{~K}$ & -0.085 & -0.016 & 0.055 & -0.104 & -0.161 & 0.001 & 0.998 \\
& $293.15 \mathrm{~K}$ & -0.080 & -0.002 & 0.075 & -0.128 & -0.184 & 0.001 & 0.998 \\
& $298.15 \mathrm{~K}$ & -0.079 & 0.012 & 0.091 & -0.136 & -0.195 & 0.000 & 0.999 \\
\hline
\end{tabular}

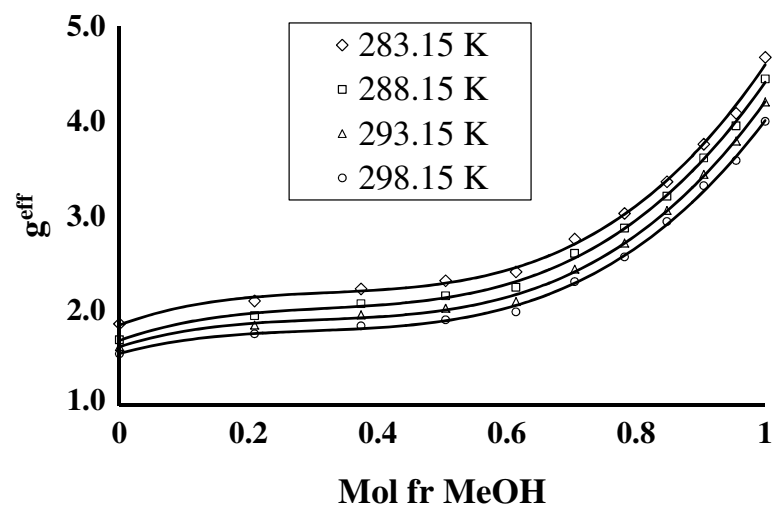

Fig. 8. Plot of effective Kirkwood correlation factor ( $\left.g^{\text {eff }}\right)$ against $\mathrm{Mol}$ fr of $\mathrm{MeOH}$. (-) solid line shows the polynomial fitted curve.

and $288.15 \mathrm{~K}$ the value of fitting parameter ' $a$ ' is positive and greater than unity, which indicate that the effective volume of the components of the binary mixtures increases on mixing, whereas less than unity values of the fitting parameter ' $a$ ' at the temperatures 293.15 and $298.15 \mathrm{~K}$ indicate that the effective volume of the components of the binary mixtures decreases on mixing. The values of fitting parameter ' $a$ ' is

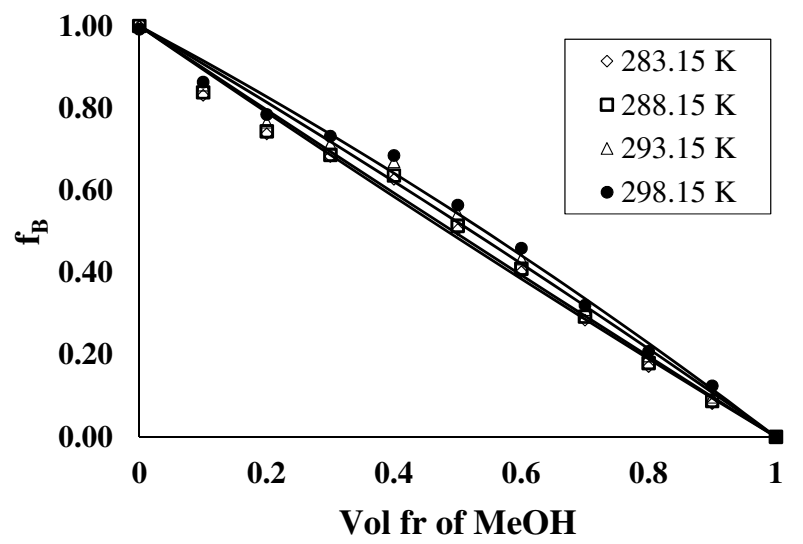

Fig. 9. Plot of Bruggeman factor $\left(f_{\mathrm{B}}\right)$ against Vol fr of MeOH. (一) solid line shows the Bruggeman equation fitted curve. higher for $\beta$-picoline $+\mathrm{MeOH}$ mixture system (Table 2) as compared to $\alpha$-picoline $+\mathrm{MeOH}$ mixture system ${ }^{7}$ at corresponding temperature, this suggests that the molecular interaction of $\mathrm{MeOH}$ is stronger with $\beta$-picoline than with $\alpha$-picoline.

Determined values of thermo dynamical parameters: molar free energy of activation $\left(\Delta F_{E}\right)$, molar enthalpy of activation $\left(\Delta H_{E}\right)$ and molar entropy of activation $\left(\Delta S_{E}\right)$ are presented in Table 3. $\Delta H_{E}$ values were determined from the slopes of the plots of $\ln (\tau T)$ versus 1000/T, which are shown in Fig. 10, while $\Delta F_{E}$ and $\Delta H_{E}$ are calculated using Eyring's rate equations. From Table 3, it can be seen that the value of free energy of activation is least for $\beta$-picoline and it increases with increase in concentration of $\mathrm{MeOH}$ at all temperatures. Higher value of $\Delta F_{E}$ of $\mathrm{MeOH}$ is due to the formation of hydrogen bonded networks in $\mathrm{MeOH}$ that tend to increase the effective size of rotating dipole and the dielectric frictional force between the rotating dipole and neighboring molecule. Furthermore, it can be seen that as the temperature increases, the molar free energy of activation for the dipole relaxation process increases for $\mathrm{MeOH}, \beta$-picoline and their mixtures. This can be attributed to the fact that as the temperature increases, thermal agitation increases and the molecules require more energy to overcome the energy barrier separating the two mean equilibrium positions. In the present study the entropy of activation $\left(\Delta S_{E}\right)$ is found to be negative for all concentrations, at all temperatures, suggesting that the orientation in the system becomes co-operative resulting in the activated state, which is less disordered than the normal state.

Table 2. Values of fitting parameter ' $a$ ' of Bruggeman factor for $\beta$-picoline + $\mathrm{MeOH}$.

\begin{tabular}{lccc}
\hline Temperature & Fitting parameter $^{a}$ & Standard error & Correction coefficient \\
\hline $283.15 \mathrm{~K}$ & 1.065 & 0.031 & 0.995 \\
$288.15 \mathrm{~K}$ & 1.026 & 0.031 & 0.995 \\
$293.15 \mathrm{~K}$ & 0.911 & 0.027 & 0.997 \\
$298.15 \mathrm{~K}$ & 0.826 & 0.03 & 0.996 \\
\hline
\end{tabular}


Table 3. Values of thermo dynamical parameters of binary mixtures of $\beta$-picoline $+\mathrm{MeOH}$ at different temperatures.

\begin{tabular}{|c|c|c|c|c|c|c|c|c|c|}
\hline \multirow[b]{2}{*}{$\mathrm{Mol} \mathrm{fr}$ of $\mathrm{MeOH}$} & \multicolumn{4}{|c|}{$\Delta F_{E}(\mathrm{~kJ} / \mathrm{mol})$} & \multicolumn{4}{|c|}{$\Delta S_{E}(\mathrm{~J} / \mathrm{mol} \mathrm{k})$} & \multirow[b]{2}{*}{$\Delta H_{E}(\mathrm{~kJ} / \mathrm{mol})$} \\
\hline & $283.15 \mathrm{~K}$ & $288.15 \mathrm{~K}$ & $293.15 \mathrm{~K}$ & $298.15 \mathrm{~K}$ & $283.15 \mathrm{~K}$ & $288.15 \mathrm{~K}$ & $293.15 \mathrm{~K}$ & $298.15 \mathrm{~K}$ & \\
\hline 0.000 & 10.045 & 10.166 & 10.303 & 10.322 & -0.028 & -0.028 & -0.028 & -0.028 & 2.112 \\
\hline 0.209 & 11.241 & 11.306 & 11.365 & 11.272 & -0.012 & -0.012 & -0.012 & -0.011 & 7.907 \\
\hline 0.374 & 11.931 & 11.948 & 11.962 & 11.895 & -0.007 & -0.007 & -0.007 & -0.006 & 10.01 \\
\hline 0.505 & 12.584 & 12.618 & 12.683 & 12.605 & -0.011 & -0.011 & -0.011 & -0.011 & 9.378 \\
\hline 0.614 & 12.892 & 12.891 & 12.935 & 12.990 & -0.015 & -0.015 & -0.015 & -0.015 & 8.547 \\
\hline 0.705 & 12.999 & 13.032 & 13.119 & 13.117 & -0.018 & -0.017 & -0.017 & -0.017 & 8.040 \\
\hline 0.782 & 13.145 & 13.147 & 13.235 & 13.246 & -0.016 & -0.016 & -0.016 & -0.016 & 8.489 \\
\hline 0.848 & 13.24 & 13.28 & 13.34 & 13.377 & -0.018 & -0.018 & -0.018 & -0.018 & 8.131 \\
\hline 0.905 & 13.337 & 13.405 & 13.438 & 13.459 & -0.017 & -0.017 & -0.016 & -0.016 & 8.622 \\
\hline 0.955 & 13.483 & 13.573 & 13.629 & 13.660 & -0.02 & -0.02 & -0.02 & -0.02 & 7.707 \\
\hline 1.000 & 13.553 & 13.644 & 13.771 & 13.819 & -0.023 & -0.023 & -0.023 & -0.022 & 7.133 \\
\hline
\end{tabular}

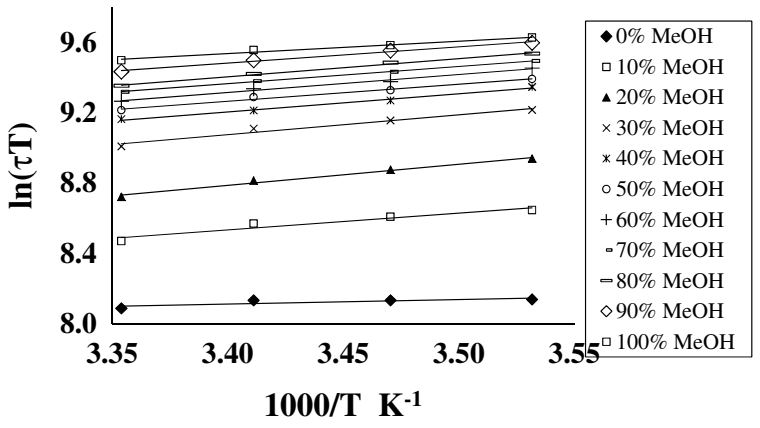

Fig. 10. Plot of $\ln (\tau T)$ versus $1000 / T \mathrm{~K}^{-1}$. (-) solid line shows trend line.

\section{Conclusion}

Complex permittivity spectra of $\beta$-picoline, $\mathrm{MeOH}$ and their mixtures display single dispersion due to main relaxation process in the frequency range of measurement at all temperatures. The plot of $\beta$-picoline exhibit the principle dispersion of Debye type where as its mixture with $\mathrm{MeOH}$ and pure $\mathrm{MeOH}$ exhibit $\mathrm{C}-\mathrm{D}$ type of relaxation behavior. The presence of additional dispersion regions is confirmed for $\beta$-picoline, $\mathrm{MeOH}$ and their mixtures by the difference in the $\varepsilon_{\infty 1}$ and $\varepsilon_{\infty}$ values. Static permittivity, permittivity at optical frequency, permittivity at high frequency, relaxation time, Kirkwood correlation factor, and Bruggeman factor and thermo dynamical parameters for $\beta$-picoline, $\mathrm{MeOH}$ and their mixtures over the entire concentration range has been determined and are reported. A nonlinear variation in static permittivity confirms that intermolecular association is taking place in the system. Hydrogen bonded molecular networks are more effectively dissociated with increase in temperature. Molecules of mixture may form multimer structures via hydrogen bonding in such a way that the effective dipole gets reduced. $(1 / \tau)^{E}$ is negative for the entire mole fraction range of $\mathrm{MeOH}$. Parallel orientation of electric dipoles has been observed in the mixture solutions of $\beta$-picoline and $\mathrm{MeOH}$.
Comparative study of binary mixtures of $\mathrm{MeOH}$ with $\alpha$-picoline and $\beta$-picoline suggests that molecular interaction of $\mathrm{MeOH}$ is stronger with $\beta$-picoline than with $\alpha$-picoline.

\section{Acknowledgments}

Financial support provided by DRS-SAP program grant [No. F. 530/10/DRS/2010 (SAP-I)] have been utilized to carry out this work and it is gratefully acknowledged. Authors are thankful to the Head, School of Physical Sciences, Swami Ramanand Teerth Marathawada University for providing laboratory facility to carry out the TDR measurement. CMT and VAR are thankful to Prof. P.N. Gajjar, Head, Department of Physics, Gujarat University, Ahmedabad for constant encouragement.

\section{References}

${ }^{1}$ V. A. Rana, A. D. Vyas and S. C. Mehrotra, Dielectric relaxation of study of mixtures of 1-propanol with aniline, 2-chloroaniline and 3-chloroaniline at different temperatures using time domain reflectometry, J. Mol. Liq. 102, 379 (2002).

${ }^{2}$ V. A. Rana, A. D. Vyas and N. M. More, Dielectric relaxation study of mixtures of mixtures of 4-flouroaniline and 1-propanol, Ind. J. Pur Appl. Phys. 40, 350 (2002).

${ }^{3}$ P. Petong, R. Pottel and U. Kaatze, Dielectric relaxation of $\mathrm{H}$-bonded liquids. Mixtures of ethanol and n-Hexanol at different compositions and temperatures, J. Phys. Chem. A 103(31), 6114 (1999).

${ }^{4}$ S. Sudo, N. Shinyashiki, Y. Kitsuki, S. Yagihara, A. C. Kumbharkhane and S. C Mehrotra, Dielectric relaxation time and relaxation time distribution of alcohol-water mixtures, J. Phy. Chem. A 106(3), 458 (2002).

${ }^{5}$ P. G. Hudge, M. P. Lokhande and A. C. Kumbharkhane, The study of dielectric relaxation in aqueous carbohydrates solutions using time domain reflectometry technique, Indian J. Phys. 86, 813 (2012).

${ }^{6}$ V. P. Pawar and S. C. Mehrotra, Dielectric relaxation study of liquids having chloro group with associated liquids. 
I. Chlorobenzene with methanol, ethanol and 1-propanol, J. Soln. Chem. 31, 559 (2002).

${ }^{7}$ C. M. Trivedi, V. A. Rana, P. G. Hudge and A. C. Kumbharkhane, Dielectric relaxation studies of binary mixture of $\alpha$-picoline and methanol using time domain reflectometry at different temperatures, J. Chinese Chem. Soc. 62, 1137 (2015), doi:10.1002/ jccs.201500396.

${ }^{8}$ P. Sivagurunathan, K. Dharmalingam, K. Ramchandran, B. Prabhakar Undre, P. W. Khirade and S.C Mehrotra, Dielectric relaxation study of mixtures of alkyl methacrylates and 1-alcohols using time-domain reflectometry, Philosophical Magazine Letters, 86(5), 291 (2006).

${ }^{9}$ B. Czech and W. Marczak, Effects of salvation of 2-Methylpyridine and 2,6-Dimethylpyridine in dilute solutions in water and methanol on the limiting partial molar compressibility and volume, Acta Phys. Pol. A 114(6-A), 45 (2008).

${ }^{10}$ B. Czech, P. Lodowski and W. Marczak, Correlation of $\mathrm{O}-\mathrm{H}-\mathrm{N}$ bonds energy with the excess compression of binary mixtures of pyridine and its methyl derivatives with methanol and water, Chem. Phys. Lett. 556, 132 (2013).

${ }^{11} \mathrm{~S}$. Ernst and W. Marczak, Hydrophobic and hydrophilic interactions in binary mixtures of $\alpha$-picoline with water, Bull. Pol. Acad. Sci. Chem. 43, 259 (1995).

${ }^{12}$ N. V. Poomachandra Rao, Studies of dielectric behaviour of picolines, Int. J. Macromol. Sci. 1(2), 23 (2011).

${ }^{13}$ U. Kaatze, C. Neumann and R. Pottel, Dielectric spectroscopy on aqueous solutions of pyridine and its derivatives, J. Soln. Chem. 16, 191 (1987).

${ }^{14}$ C. E. Shannon, Communication in the presence of noise, Proc. IRE 37, 10 (1949).

${ }^{15}$ H. A. Samulon, Spectrum analysis of transient response curves, Proc. IRE 39, 175 (1951).

${ }^{16} \mathrm{R}$. Buchner and J. Barthel, Dynamics of methanol-tetra chloromethane mixtures- $\alpha$ dielectric relaxation study, J. Mol. Liq. 52, 131 (1992).

${ }^{17}$ D. W. Davidson and R. H. Cole, Dielectric relaxation in Glycerol, propylene glycol and n-propanol, J. Chem. Phys. 19, 1484 (1951).
${ }^{18}$ P. Debye, Polar Molecules (Chemical Catalog Company, NewYork 1929).

${ }^{19}$ R. MacDonald, LEVM/ LEVMW Manual. Issue 8.0 (2014).

${ }^{20}$ J. Z. Bao, M. L. Swicord and C. C. Davis, Microwave dielectric characterization of binary mixtures of water, methanol and ethanol, J. Chem. Phys. 104, 4441 (1996).

${ }^{21}$ J. G. Kirkwood, The dielectric polarization of polar liquids, $J$. Chem. Phys. 7, 911 (1939).

${ }^{22}$ A. C Kumbharkhane, S. M. Puranik and S. C. Mehrotra, Dielectric relaxation studies of aqueous $\mathrm{N}, \mathrm{N}$-dimethylformamide using a picosecond time domain technique, J. Soln. Chem. 22, 219 (1993).

${ }^{23}$ G. Moumouzias, D. K. Panopoulos and G. Ritzoulls, Excess properties of the system propylene carbonate + acetonitrile, $J$. Chem. Eng. Data 36, 20 (1991).

${ }^{24}$ S. C. Mehrotra and J. E. Broggs, Effect of collision-induced phase shifts on the line widths and line shifts of rotational spectral lines, J. Chem. Phys. 66, 5306 (1977).

${ }^{25}$ D. A. G. Bruggeman, Calculation of various physical constants of heterogeneous substances. Dielectric constants and conductivities of the mixed body of isotropic substances, Ann. Phys. 5, 636 (1935).

${ }^{26}$ H. Frohilich, Theory of Dielectrics (Oxford University Press, London, 1949).

${ }^{27}$ S. Glasston, K. Laider and H. Erying, The Theory of Rate Processes (Mc Grew Hill Book Co., New York, 1941), pp 548-553.

${ }^{28}$ O. Redlich and A. T. Kister, Algebraic representation of thermodynamic properties and the classification of solutions, Ind. Eng. Chem. 140, 345 (1948).

${ }^{29}$ W. D. Callister Jr., Materials Science and Engineering An Introduction, 6th Edn., (Wiley-India Edi., 2007), pp. 711-723.

${ }^{30}$ L. C. Wang, H. S. Xu, J. H. Zhao, C. Y. Song and F. Wang, Density and viscosity of (3-picoline+water) binary mixtures from $\mathrm{T}=(293.15$ to 343.15$) \mathrm{K}$, J. Chem. Thermo. 37, 477 (2005).

${ }^{31}$ H. Freiser, W. L. Glowacki, Some physical properties of 2-picoline, J. Am. Chem. Soc. 70, 2575 (1948). 